\title{
Memory for lateral asymmetries in well-known faces: Evidence for configural information in memory representations of faces
}

\author{
GILLIAN RHODES \\ Stanford University, Stanford, California
}

\begin{abstract}
Two experiments were carried out to determine whether configural information about the left-right organization of a face is represented in memory. In Experiment 1, subjects consistently chose normal (correct) photographs as better likenesses of familiar faces than mirror-reversed photographs. This effect was stronger for more familiar faces, and did not depend on the presence of asymmetric hairstyles or single, asymmetrically located features (e.g., moles, warts). In Experiment 2, subjects were asked to decide which of the left and right composite faces was a better likeness of the person. The left composite is normally considered a better likeness in perceptual matching tasks where the normal photograph is present. Left composites were regarded as better likenesses than right composites, despite the fact that this bias appeared to compete with one in favor of the more realistic of the two composites. The results of Experiments 1 and 2 suggest that configural information about the left-right organization of faces is represented in memory. The right hemiface of a familiar face was considered more expressive than the left hemiface. This is opposite to the normal result for unfamiliar faces. The implications of the results for models of face representations and for future research directions are discussed.
\end{abstract}

Despite the similarity of faces as visual patterns, we are able to discriminate between and remember many faces for long periods of time (Bahrick, Bahrick, \& Wittlinger, 1975). Bahrick et al. showed that people could recognize pictures of classmates at a $90 \%$ correct performance level, independent of class size, for up to 35 years after graduation. Our ability to remember faces, often over an extended period of time, may be essentially unlimited under natural conditions of learning with repeated, long duration exposures to a face. Even a single, brief exposure may be sufficient to encode a face in memory. Faces signal identity and emotional state, both of which are important predictors of behavior. Therefore, there has probably been strong selection pressure for the ability to encode faces quickly and to remember them for long periods of time.

Our capacity for encoding and remembering faces is not yet understood, and theories of facial representation are not well developed. Past research has emphasized the importance of individual features, such as the eyes, nose, and mouth, for discriminating between and remembering faces. Changing these features impairs discrimination and recognition performance (for a comprehensive review, see Shepherd, Davies, \& Ellis, 1981). Winograd

This research was supported by NSF Grant BNS 80-05517 to Roger Shepard at Stanford University. I would like to thank Ian McLean for taking the photographs used in these experiments, Gloria Johnson and Katy Babcock for helping with the data collection, and everyone who allowed their photographs to be taken and used in the experiments. I also thank Ann O'Leary for helpful discussions about this work. Requests for reprints should be sent to G. Rhodes, Department of Psychology, Otago University, P.O. Box 56, Dunedin, New Zealand.
$(1978,1981)$ proposed that faces are encoded as sets of features. This proposal is based on the effects of encoding instructions on memory for faces. Instructions to make trait judgments (e.g., honesty, friendliness, likability or expressiveness), which are assumed to induce "deep" or semantic processing, produced better memory for faces than did instructions to make physical feature judgments (Bower \& Karlin, 1974; Warrington \& Ackroyd, 1975). These are the effects predicted by Craik and Lockhart's levels-of-processing theory (Craik \& Lockhart, 1972). However, if subjects were asked to scan for the most distinctive feature of a face, memory was as good as with trait instructions (Courtois \& Mueller, 1979; Winograd, 1981). Furthermore, Winograd demonstrated that judgments about a single feature produced recognition performance as good as with trait judgments, if that feature was a distinctive one (as determined by subjects' ratings). In fact, trait judgments only enhanced memory for faces that had a distinctive feature. These results led Winograd to propose that trait judgments enhance memory only to the extent that they induce more features of a face to be encoded (the elaboration hypothesis), and that elaboration is only helpful to the extent that encoding more features increases the likelihood of encoding a distinctive feature. He concluded that faces are encoded in terms of features and that the presence of a distinctive feature facilitates memory. ${ }^{1}$

There is, however, another aspect of faces that may be important for individuation and recognition. This is the spatial relationship between the features, that is, configural information, such as distances and ratios of distances between features. Configural information is sufficient to identify very blurred faces, in which no details about the 
appearance of features are available (Harmon, 1973). Moreover, training people to analyze faces in terms of component features seems to impair recognition (Baddeley, 1979), which suggests that faces may not normally be encoded as feature sets. Recent studies (Sergent, 1984a, 1984b) offer support for the importance of configural information in the perception of schematic faces. Sergent (1984a) fitted several models of how component features are combined to same-different reaction time data obtained for pairs of schematic faces that varied factorially on three dimensions. The results indicated that features were processed interactively and that configural information about the relationship between features was used to make the judgments. Dissimilarity judgments about pairs of schematic faces were also shown to depend on configural information in the faces (Sergent, 1984b).

A model of face representations as sets of features, with no information about the spatial relations between features, also seems unrealistic, based on what is known about the neuropsychology of face recognition. Face perception and recognition is mediated more efficiently by the right than the left hemisphere, and a feature-based strategy appears to be quite atypical (see Rhodes, 1985a, for a review). Studies with schematic faces have shown that there is a right hemisphere advantage for face recognition only if target faces are defined in terms of feature configurations, and not if they are defined in terms of single features (Bradshaw \& Sherlock, 1982). Recognition of faces presented to the right hemisphere of split-brain patients is good, and appears to be based on recognition of the entire facial configuration (Levy, Trevarthen, \& Sperry, 1972). Split-brain patients recognize faces presented to the left hemisphere by examining features that are easily labeled verbally, a strategy that leads to poor recognition performance. These results suggest that faces are not normally encoded simply as sets of features. Configural information about the spatial relations between features also appears to be represented.

Experiment 1 was designed to test the hypothesis that configural information about the left-right organization of a face is represented in memory. This information could be about the vertical position of one eye relative to the other, the direction in which a crooked nose leans, asymmetries in the bone structure of the two sides of the face, and so forth. The hypothesis is that we represent configural information about a face in memory. The experiment does not directly address the issue of how this information is represented, although the kind of information represented has implications for the structure of the representation (see General Discussion). Subjects were asked which of the normal and mirror-reversed photographs of a familiar face was a better likeness of the person. If configural information about the left-right organization of faces is represented, then subjects should choose the normal images. There is evidence that under typical experimental learning conditions of brief exposure to many faces, subjects encode information about the lateral orientation of faces. McKelvie (1983) found that mirror reversal of photographs of faces between study and test, separated by $10 \mathrm{~min}$, reduced recognition significantly for both percent correct and $d^{\prime}$. However, the same photographs were used at study and test, so subjects may have based their judgments on aspects of the photographs other than the identity of the face (e.g., irrelevant marks on the slides). Mita, Dermer, and Knight (1977) found that people preferred normal to mirror-reversed photographs of the faces of close friends or lovers. This indicates that people can discriminate between the two views, although the study does not show that people know which is the correct (normal) view. Experiment 1 directly tests the hypothesis that people know which is the normal orientation of a face that has been encoded under naturalistic conditions.

In Experiment 2, subjects were asked to make another discrimination that requires configural information about the left-right organization of a face. They were asked whether the left or right composite was a better likeness of the person. ${ }^{2}$ The left composite is considered a better likeness of the face in perceptual matching tasks where the normal photograph of an unfamiliar face is present (Gilbert \& Bakan, 1973; Kolb, Milner, \& Taylor, 1983; Lindzey, Prince, \& Wright, 1952; McCurdy, 1949; Rhodes, 1985b; Wolff, 1933). It is not clear whether this effect is due to direct access of the left half of the face to the right hemisphere (which assumes that the left halfface $^{3}$ lies mostly in the left visual field) or whether it is due to asymmetric scanning or direction of attention to the face (see Rhodes, 1985b, for a discussion of these possibilities). Whatever the mechanism, the phenomenon indicates that the left half of the face dominates the perceptual representation, and in this sense, configural information about the left-right asymmetry of the face is encoded. If this asymmetry is maintained in the memory representation of the face, then subjects should choose the left composite as a better likeness of a familiar person (in the absence of a veridical photograph).

Assymmetries in the perceived expressiveness of the two sides of familiar faces were also examined in these experiments, to determine whether familiarity with a face affects the perceived asymmetry in expressiveness. For unfamiliar faces, there is evidence that the left hemiface is perceived to be more expressive than the right hemiface (for a review, see Bryden \& Ley, 1983). This is called facedness and reflects the greater involvement of the right hemisphere (which controls muscles in the lower twothirds of the left hemiface) in the production of emotional expressions (Brodal, 1965). The results of these studies should indicate whether the expressiveness of familiar faces is perceived in the same way.

\section{EXPERIMENT 1}

Experiment 1 was designed to test the hypothesis that information about the lateral organization of faces, including one's own face, is available in naturally acquired memory representations of faces. Subjects were asked to 
decide which of the normal and mirror-reversed pictures of well-known faces and of their own faces were better likenesses of the people. Both neutral and smiling photographs were used. Subjects were asked to judge which of the normal and mirror-reversed smiling faces looked happier. Because the side of the face appearing on the observer's left dominates what the face looks like (Gilbert \& Bakan, 1973; Lindzey et al., 1952; McCurdy, 1949; Rhodes, 1985b; Wolff, 1933) ${ }^{4}$ and what emotion is perceived (Campbell, 1978; Heller \& Levy, 1981), it was predicted that the smiling mirror-reversed faces, with the more expressive left hemiface on the observer's left, should look happier than the smiling normal faces.

\section{Method}

\section{Subjects}

Twenty-two subjects did the mirror-image discrimination task. Seventeen of these, plus the 10 additional people whose photographs had been taken, did the self-perception task ( 27 subjects). Twentytwo Stanford undergraduates, who were unfamiliar with the faces, participated in a control experiment (see below).

\section{Stimuli}

Sixteen women and 11 men were photographed. All were students, staff, or faculty members of the Stanford University Psychology Department. The only selection criterion for these volunteers was that the males should not have beards. People who normally wore glasses were photographed without them. The two sides of the face were evenly illuminated.

Several full-face, black-and-white photographs were taken of each person, some smiling and some with a neutral expression. Smiles were elicited during the course of conversation with the photographer, who joked with the volunteers. Two independent raters agreed on the expression (neutral or smiling) displayed in each photograph, and one neutral and one smiling photograph were chosen for each person. The best 20 picture pairs ( 10 female and 10 male) were chosen for use in the mirror-image discrimination and happiness judgments parts of the experiment (see below). ${ }^{5}$ The remaining 7 picture pairs (6 female and 1 male) were used in the selfperception test only (see below).

Normal and mirror-reversed prints were obtained for each picture. All pictures measured $3.5 \times 5$ in. For each of the 20 faces with neutral expressions, the normal and mirror-reversed prints were arranged vertically on a page. Half the time the normal print was the top one, and half the time the reversed print was the top one. These pages were then assembled into an album in random order. The same order was used throughout the experiment. A similar album was made for the smiling pictures. These two albums were used for the mirror-image discrimination part of the experiment. Picture pairs for the remaining 7 people were also arranged vertically on separate pages for use in the self-perception part of the study. The normal print was the top one in four of these picture pairs. Pictures of all 27 people were used in the self-perception test.

\section{Procedure}

Self-perception test. The neutral pair was shown first, and each subject was asked to decide which photograph was a better likeness of himself/herself. Then they were shown the smiling pair and asked the same question. Finally, they were asked which of the two smiling pictures looked happier.

Mirror-image discrimination test. The self-perception test was completed first if both tasks were done. Subjects were asked to decide which photograph of each normal and mirror-reversed pair was a better likeness of the person. They were encouraged to base their judgments on first impressions. The smiling photographs were judged after the neutral faces so that the subjects' attention would not be focused on expression when judging the neutral pairs. Subjects indicated, on a 5-point rating scale, how familiar they were with each person's face $(1=$ never seen the face before, $5=$ highly familiar). They also rated, on a 10-point scale, how similar the two neutral photographs looked $(1=$ not at all similar, $10=$ identical $)$.

Happiness judgments. After completing the mirror-image discrimination task, subjects decided which of each pair of the smiling faces looked happier, and rated how similar the expressions in the two faces looked on a 10-point scale.

Control experiment. A control experiment was carried out after the data from Experiment 1 had been analyzed, in order to help interpret those results. Different subjects, who were unfamiliar with the faces, were asked which face in each pair of smiling faces looked happier. These data provided a baseline against which to assess the effect of familiarity on the perceived relative happiness of the normal and mirror-reversed pictures.

\section{Self-Perception}

For the pair of photographs showing a neutral expression, significantly more subjects considered the mirrorreversed picture to be a better likeness of themselves $(N=19)$ than the normal picture $(N=8)$, as predicted $\left[\chi^{2}(1)=4.48, p<.025\right.$, one-tailed $]$. This was not the picture that others thought looked more like the person (see below), supporting the commonsense notion that the representation of one's own face comes from looking in mirrors, at least for neutral expressions. For smiling faces, there was no consistent preference. Thirteen subjects chose the mirror-reversed picture and 14 chose the normal picture as a better likeness of themselves $\left[\chi^{2}(1)=\right.$ $.02, p>.05$ ]. It is possible that each subject had a representation of his/her face based on photographs which competed with the more familiar mirror-image representation. That the normal (photographic) representations should be stronger than the mirror-image representations for the smiling but not the neutral photographs might reflect the fact that people are usually smiling in photographs, but adopt a neutral expression when looking in mirrors. Alternatively, people may be relatively unfamiliar with their smiling faces, and so may choose randomly between the normal and mirror-reversed smiling images.

As predicted by the facedness hypothesis, more subjects chose the mirror-reversed $(N=16)$ than the normal photograph of themselves $(N=11)$ as looking happier. However, this difference was not significant $\left[\chi^{2}(1)=.93\right.$, $p>.05]$.

\section{Mirror-Image Discrimination Test}

Scores of the proportion of normal photographs chosen (i.e., correct responses) were obtained for each subject. Separate scores were obtained for judgments about the neutral expression faces and the smiling faces, at each of the four levels of rated familiarity (2-5, higher numbers indicate greater familiarity). Data from faces that the subjects had never seen before (rated 1 on the 5-point scale) were excluded. The mean number of such faces for each subject was .5 out of 20 faces. The four familiarity levels were collapsed into a low- and a high-familiarity group. ${ }^{6}$ Scores did not differ for faces in the groups that were col- 
lapsed across [mean $=.58$, faces rated two; mean $=.60$, faces rated three; $F(1,63)<1$; mean $=.72$, faces rated 4 ; mean $=.73$, faces rated $5 ; F(1,63)<1$ ]. Nor was there any interaction between familiarity and expression type $[F(3,63)=2.34, p>.05]$. The terms low and high should be interpreted as indicating relative rather than absolute levels of familiarity. Mean scores for the low- and high-familiarity categories for the neutral and smiling photographs are shown in Table 1.

A two-way analysis of variance was carried out with familiarity and expression type as within-subject factors. The main result of interest was that, as predicted, the overall proportion of correct responses (mean $=.66$ ) was significantly better than chance [mean $=.50 ; F(1,21)=$ $13.14, p<.005$, one-tailed]. There was a significant effect of familiarity $[F(1,21)=18.92, p<.0001]$, with better performance for more familiar faces (mean $=.73$ ) than for less familiar faces (mean $=.59$ ). Considered separately, performance was significantly better than chance for both the highly familiar faces $[F(1,21)=27.50$, $p<.00005$, one-tailed] and for the less familiar faces $[F(1,21)=4.02, p<.05$, one-tailed $]$. The fact that the effect was larger for more familiar faces supports the idea that the task was done by comparing the photographs to memory representations of the faces. The memory representations of more familiar faces are presumably more accessible or more elaborated than those of less familiar faces, thereby allowing the forward and mirrorreversed photographs to be more reliably discriminated. The larger effect for the more familiar faces cannot be accounted for by differences in the stimuli because different stimuli were in the low- and high-familiarity groups for different subjects. In addition, subjects did not rate the similarity of the two neutral photographs in each pair of faces differently for the high- $($ mean $=7.3)$ and lowfamiliarity sets [mean $=7.4 ; F(1,21)<1]$. There was no significant effect of expression on proportion of correct responses $[F(1,21)<1]$. Nor was there any interaction between familiarity and expression $[F(1,21)<1]$.

In order to ensure that these results are generalizable across faces, an analysis of variance was carried out with faces as the random factor and familiarity and expression type as within-face factors. ${ }^{7}$ The dependent variable was the proportion of subjects who chose the correct face for each pair of photographs of the same face (see Table 2). The pattern of results was the same as that obtained with subjects as the random factor (see above). The mean proportion of people choosing the correct photograph for each face was .68 , which was significantly better than

Table 1

Mean Proportion of Normal (Correct) Faces Chosen as Better Likenesses (Experiment 1)

\begin{tabular}{lcccc}
\hline & \multicolumn{4}{c}{ Familiarity } \\
\cline { 2 - 5 } Expression & Low (Rated 2 or 3) & \multicolumn{2}{l}{ High (Rated 4 or 5) } \\
\cline { 2 - 5 } \cline { 3 - 5 } Nean & SE & & Mean & SE \\
\hline Smiling & .58 & .03 & .72 & .04 \\
\hline
\end{tabular}

Table 2

Mean Proportion of Subjects Choosing the Normal (Correct) Face as a Better Likeness for Each Face (Experiment 1)

\begin{tabular}{lccccc}
\hline & \multicolumn{4}{c}{ Familiarity } \\
\cline { 2 - 5 } Expression & Low (Rated 2 or 3) & & \multicolumn{2}{c}{ High (Rated 4 or 5) } \\
\cline { 2 - 3 } \cline { 5 - 6 } & Mean & SE & & Mean & SE \\
\hline Neutral & .60 & .05 & .79 & .03 \\
Smiling & .59 & .05 & .74 & .04 \\
\hline
\end{tabular}

chance $(.50)[F(1,19)=13.51, p<.005$, one-tailed $]$. More than half the subjects chose the correct photograph for 17 of the 20 faces used. There was a significant effect of familiarity $[F(1,19)=16.46, p<.001]$, with more subjects choosing the correct face for highly familiar faces (mean $=.76$ ) than for less familiar faces (mean $=$ .60 ). As in the previous analysis, performance was better than chance for both the high- and low-familiarity faces considered separately $[F(1,19)=29.16, p<.00005$, one-tailed (high); $F(1,19)=3.81, p<.05$, one-tailed (low)]. There was no effect of expression type $[F(1,19)<1]$. Nor was there any interaction between familiarity and expression type $[F(1,19)<1]$. These results indicate that the ability to discriminate between normal and mirror-reversed familiar faces generalizes across faces.

It is possible that people could have based their judgments on asymmetries in the hairstyles, rather than in the faces themselves. This possibility could have been eliminated by hiding the hair of the posers (e.g., by having them wear a scarf) or by masking the hair in the photographs. Neither of these approaches was used, because it was considered important that the faces look as normal as possible. Instead, the effect of asymmetric hairstyles was partialled out after the data were collected. Because less than half (9) of the 20 posers had asymmetric hairstyles, ${ }^{8}$ and because the ANOVA with faces as a random factor indicated that the results generalized across faces, it seemed unlikely that subjects were basing their judgments solely on asymmetries in the hairstyles. However, a further check of this was carried out. Separate scores were obtained for each subject, for posers with symmetric hairstyles and for those with asymmetric hairstyles. These were subjected to a two-way analysis of variance, with expression type and symmetry of hairstyle as withinsubject factors. There was a significant effect of symmetry of hairstyle $[F(1,21)=5.47, p<.05]$, with better performance for faces with asymmetric hairstyles (mean = .72) than for those with symmetric hairstyles (mean $=$ .64). However, performance was still well above chance when the hairstyles were symmetric $[F(1,21)=8.52$, $p<.01]$. There was no significant effect of expression $[F(1,21)<1]$. The interaction between expression and symmetry of hairstyle was not significant $[F(1,21)=$ $3.55, p<.07$ ], although there was a trend for the effect of symmetry to be larger for neutral faces (mean $=.76$, asymmetric; mean $=.63$, symmetric) than for smiling faces (mean $=.69$, asymmetric; mean $=.66$, symmetric). These results indicate that although subjects did use 
asymmetries in the hairstyles to make discriminations, they could select the correct faces much better than chance in the absence of such information.

The discriminations could also have been based on the location of single, distinctive, asymmetric features such as moles or warts. The location of an asymmetrically placed feature is itself configural information, because position must be coded relative to other parts of the face. In order to determine whether this type of configural information was being used, performance was compared for faces with and without such features. Four independent judges selected faces that had permanent, asymmetrically located features (transient features, such as pimples, were excluded from consideration). Faces were considered to have these features if they were selected by at least 2 of the judges. An analysis of variance with expression type and face type (with or without single, asymmetrically placed features) as within-subject factors was carried out. There was no effect of face type $[F(1,21)<1$; mean $=.67$ for faces with asymmetric features, $N=7$; mean $=.68$ for faces without asymmetric features, $N=13]$. There was no effect of expression type $[F(1,21)<1$; mean $=.67$, neutral faces; mean $=.67$, smiling faces] and no interaction between expression and face type $[F(1,21)=2.02, p>.05]$. Nor was there any effect of face type when performance for faces with either asymmetric hairstyles or single, asymmetrically located features (mean $=.69,13$ faces) was compared with performance for faces without either (mean $=.65,7$ faces) $[F(1,21)=1.49, p>.05]$.

The results of Experiment 1 indicate that subtle configural information about the left-right organization of well-known faces is available in memory. Normal and mirror-reversed faces can be correctly discriminated even in the absence of single, asymmetrically located features (e.g., moles or warts) or asymmetric hairstyles.

\section{Happiness Judgments}

An analysis of variance was carried out with familiarity as a within-subject factor. Contrary to expectation, subjects judged the normal photographs to look happier than the mirror-reversed photographs. The mean proportion of normal photographs chosen as looking happier was .60, which was significantly greater than $.50[F(1,21)=6.92$, $p<.05]$. Subjects chose the normal photographs significantly more often for the highly familiar faces (mean = .68) than for the less familiar faces [mean $=.53$; $F(1,21)=10.34, p<.01]$. Considered separately, normal faces were chosen more often than chance only for the highly familiar faces $[F(1,21)=20.89, p<.0001]$. For the less familiar faces, the normal and mirror-reversed photographs were not differentially chosen $[F(1,21)<1]$.

In order to determine whether these results generalize across faces, an analysis of variance was carried out with faces as the random factor and familiarity as a withinface factor. The dependent variable was the proportion of subjects choosing the normal photograph as happier for each face. The mean proportion of subjects choosing the normal photograph as happier for each face was .59 , which was significantly greater than $.50[F(1,19)=5.44$, $p<.05]$. The mean proportion of subjects choosing the normal face was greater for the highly familiar faces (mean $=.65$ ) than for the less familiar faces (mean $=$ .53 ), although the effect was only marginally significant $[F(1,19)=4.19, p=.052]$. Considered separately, normal faces were selected as happier by more subjects for the highly familiar faces $[F(1,19)=14.37, p<.01]$, but not for the less familiar faces $[F(1,19)<1]$. For the highly familiar faces, the proportion of subjects choosing the normal photograph as happier was greater than .50 for all 20 faces. These results are consistent with those from the analysis with subjects as a random factor. Together they indicate that for highly familiar faces, the normal photographs look happier than the mirror-reversed photographs. For less familiar faces, the normal and mirror-reversed faces look equally happy.

The result that the normal photographs looked happier than the mirror-reversed photographs was unexpected. It was predicted that the mirror-reversed faces, with the more expressive left hemiface appearing on the left (the side which dominates the impression of what the face looks like and what expression it displays) would look happier. It is possible that the lateral organization of a very familiar face is so strongly encoded that the reversed photographs look odd, and that this is inconsistent with their looking happy. In this case, the normal photographs would not be expected to look happier if the faces were unfamiliar. This hypothesis was tested in a control experiment.

Control results. Subjects who were unfamiliar with the faces were asked which of each pair of smiling faces looked happier. The mean proportion of normal photographs chosen was .49 , which did not differ significantly from $.50[t(21)<1]$. Therefore, when the faces were completely unfamiliar (or only moderately familiar), the normal and mirror-reversed photographs looked equally happy. This supports the interpretation advanced above that the normal faces looked happier because the images were familiar. Therefore, these results are not relevant to the question of which side of the face looks more expressive.

\section{EXPERIMENT 2}

Experiment 2 was designed to determine whether the perceptual asymmetry in favor of the left composite's looking more like the face (Gilbert \& Bakan, 1973; Kolb et al., 1983; Lindzey et al., 1952; Rhodes, 1985b; Wolff, 1933 ) is maintained in memory. Subjects were asked to decide whether the left or right composite face was a better likeness of the person. If the left composites are chosen as looking more like the faces, then this indicates that the left half of the face is more strongly represented in memory, or more accessible, than the right half of the 
face. In this case, the two sides of the face could not be represented equally in memory, and in this sense, an asymmetry between them would be encoded.

Unlike the discrimination required in Experiment 1, the decision about which composite looks more like the face cannot be based on the location of single, asymmetrically placed features, because these either occur on both sides of the image or are completely absent. Therefore the predicted result, together with the results of Experiment 1, would provide converging evidence that subtle configural information, other than the location of asymmetrically located features, is represented in memory representations of faces.

A direct test of whether the left or right hemiface is perceived as expressing more emotion in familiar faces was carried out. Subjects were asked to decide whether the left or right composite face showed more emotion, and to rate the valence of the emotion in that face, on a happy-sad scale.

\section{Method}

\section{Subjects}

Twenty-four subjects did the composites task. Fourteen of these did the self-perception task, together with the 13 other people whose photographs were available ( 27 subjects, all of whom had been subjects in the self-perception part of Experiment 1). Fourteen of the 24 subjects who did the composites task had done the mirror-image discrimination test and happiness judgments in Experiment 1 several months earlier. Twenty-four Stanford undergraduates, who were unfamiliar with the faces, participated in a control experiment (see below).

\section{Stimuli}

The same photographs were used as in Experiment 1. For each face, the vertical midline was defined as the line passing through the midpoint of a line between the two internal canthi of the eyes and through the central vertex of the upper lip. The normal and reversed prints were cut along this midline, and opposite halves joined, resulting in symmetric left and right composite photographs for each face. The left composite was made from the right hemiface, which normally appears on the left of the face from an observer's viewpoint. Similarly, the right composite was made from the left hemiface. The left and right composites for each face were arranged vertically on a page. Half the time the left composite was on top, and half the time it was on the bottom. As in Experiment 1, the pages were assembled randomly into two booklets, one containing neutral faces, and the other containing smiling faces. Pages with the left and right composites of the remaining 7 people were used for self-judgments only. The left composite was on the top for four of the seven picture pairs.

\section{Procedure}

Self-perception task. Each subject was asked to choose which of the two composites was a better likeness of himself/herself. The composites were referred to as faces, and subjects were not told how they had been constructed. Fourteen subjects judged the neutral pair first and 13 judged the smiling pair first. For each pair, subjects then chose the composite that showed more emotion, and rated that emotion on a 7-point scale ranging from sad (1) to happy (7). As with the likeness judgments, 14 subjects judged the neutral faces first and 13 judged the smiling faces first. The order of the neutral and smiling faces was counterbalanced for the likeness and emotion judgments. (Because there were 27 subjects, one of the four possible orders had 1 less subject than the other three.) Selfjudgments were made before the likeness judgments, if both tasks were done (except for 1 subject, who was not given her own photographs first, due to an oversight).

Likeness judgments. Subjects were instructed to choose which of each pair of composite faces was a better likeness of the person. They also rated how familiar they were with each face on a 5-point scale $(1=$ not at all familiar, $5=$ very familiar $)$ and how similar the two faces were on a 10-point scale $(1=$ not at all similar, 10 $=$ identical) . Similarity ratings were collected to determine whether the familiarity of a face had any effect on how similar the two composites looked. It is possible that more subtle differences between the two hemifaces can be perceived in very familiar faces. Subjects were encouraged to give their first impressions, and to try to ignore strange hairstyles or any differences in the widths of the two faces. (These instructions were also given for the selfjudgments.) Half the subjects judged the neutral faces first, and half judged the smiling faces first.

Emotion judgments. Subjects were asked to choose which composite expressed more emotion, and to rate the emotion of that face on a 7 -point scale $(1=\operatorname{sad}, 7=$ happy $)$. This was always done after the composites task, so that the likeness judgments would be independent of the emotion judgments. Making the emotion judgments first could draw attention to the expressions of the faces and bias subjects against choosing the left composites (made from the right hemifaces), if expressions were stronger on the left hemiface. Half the subjects judged the neutral faces first, and the other half judged the smiling faces first. This was counterbalanced with the order in which likeness judgments were made for the neutral and smiling faces.

Control experiment. A control experiment was carried out after the data from Experiment 2 were analyzed, in order to help interpret those results. Subjects who were unfamiliar with the faces? were asked to choose which of each pair of composites looked more like a real face. This was done to ensure that the bias in favor of left composites found with familiar faces (see below) did not result from some artifact of the stimuli that made the right composites look strange. It was also hoped that these data would be useful in determining which subset of faces yielded the bias (the effect did not generalize across faces). The control subjects were then asked which composite expressed more emotion. This was done to determine whether the usual finding, that the right composite looks more expressive, could be replicated with these stimuli. This was necessary in order to ensure that the unexpected bias in favor of left composites of familiar faces' looking more expressive (see below) was not due to anything unusual about the expressiveness of these stimuli.

\section{Results and Discussion}

\section{Self-Perception}

For the neutral photographs, 14 subjects chose the right composite and 13 chose the left composite as looking more like themselves $\left(\chi^{2}=.04, p>.05\right)$. For the smiling photographs, 13 chose the right composite and 14 chose the left composite as looking more like themselves $\left(\chi^{2}=.04\right.$, $p>.05)$. These results are consistent with the results of Experiment 1 for the smiling faces but not for the neutral faces. In Experiment 1, subjects reliably chose the mirror-reversed neutral face as looking more like themselves. Therefore, the right composite should have looked more like themselves because it was constructed from the left hemiface, which appears on the left in the mirror image. ${ }^{10}$ However, there was no asymmetry in the selfperception judgments in Experiment 2, for either the neutral or the smiling faces. It should be noted that neither composite had been seen before, and that even the ability to distinguish between the more familiar normal and mirror images of one's own face was not overwhelmingly 
robust (it was found for neutral but not for smiling photographs).

For the neutral photographs, 15 subjects chose the right and 12 subjects chose the left composite as expressing more emotion $\left(\chi^{2}=.33, p>.05\right)$. For the smiling faces, 14 subjects chose the right composite and 13 chose the left composite $\left(\chi^{2}=.04, p>.05\right)$. Thus, there was no asymmetry in perceived expressiveness of the two halves of subjects' own faces. The mean ratings of expression for the composites chosen as more expressive were 3.7 $(\mathrm{SD}=.9)$ for the neutral photographs and $6.3(\mathrm{SD}=.6)$ for the smiling photographs $(1=\mathrm{sad}, 7=$ happy $)$. These results parallel those of Experiment 1, where the forward and mirror-reversed photographs were considered equally expressive.

The self-perception judgments of Experiments 1 and 2 suggest either that there is little left-right differentiation in the memory representation of one's own face, or that there are two representations-one based on looking in mirrors and the other based on seeing photographs of oneself. The existence of both a forward and a mirrorreversed representation would account for the general inability to discriminate between forward and mirrorreversed images of one's own face (except for the neutral faces in Experiment 1, which probably resulted from the neutral mirror image being more familiar than the neutral forward image).

\section{Likeness Judgments}

Scores of the proportion of left composites chosen were calculated for each cell of the design for each subject (see Table 3). ${ }^{11} \mathrm{~A}$ three-way analysis of variance was carried out with expression (neutral or smiling) and familiarity level (low or high) as within-subject factors and order of the booklets (neutral then smiling faces or smiling then neutral faces) as a between-subjects factor. The overall mean proportion of left composites chosen was .63, which was significantly higher than chance (.50), as predicted $[F(1,22)=4.83, p<.025$, one-tailed $]$. Thus subjects judged left composites to look more like people's faces than right composites. This supports the hypothesis that the perceptual asymmetry in favor of the half-face on the viewer's left is maintained in the memory representation. There were no significant effects of order $[F(1,22)<1]$, expression $[F(1,22)<1]$, or familiarity $[F(1,22)<1]$. There was a significant interaction between expression

Table 3

Mean Proportion of Left Composites Chosen as Better Likenesses (Experiment 2)

\begin{tabular}{|c|c|c|c|c|c|c|}
\hline \multirow[b]{3}{*}{ Expression } & \multicolumn{6}{|c|}{ Familiarity } \\
\hline & \multicolumn{2}{|c|}{ Unfamiliar } & \multicolumn{2}{|c|}{ Low (Rated 2 or 3 ) } & \multicolumn{2}{|c|}{ High (Rated 4 or 5} \\
\hline & Mean & SE & Mean & SE & Mean & $\mathrm{SE}$ \\
\hline Neutral & .39 & .02 & .67 & .05 & .61 & .06 \\
\hline Smiling & .39 & .03 & .59 & .03 & .64 & .02 \\
\hline
\end{tabular}

Note-Data for the unfamiliar faces are from different subjects than data for the low- and high-familiarity faces. Subjects who were unfamiliar with the faces were asked which (composite) face looked more like a real face. and familiarity $[F(1,22)=5.26, p<.03]$. For lowfamiliarity faces, a higher proportion of left composites was chosen for the neutral faces (mean $=.67$ ) than for the smiling faces (mean $=.61$ ). For high-familiarity faces, the reverse pattern occurred, with more left composites chosen for the smiling (mean $=.64$ ) than for the neutral faces (mean $=.59$ ). However, none of these differences was significant by tests of simple main effects. Order did not interact with expression $[F(1,22)=1.44$, $p>.05]$ or familiarity $[F(1,22)<1]$. Nor was the threeway interaction between order, expression, and familiarity significant $[F(1,22)=1.33, p>.05]$.

A three-way analysis of variance was carried out on the rated similarity of the left and right composites to each other, with order as a between-subjects factor, and expression and familiarity as within-subject factors. There was a significant effect of familiarity $[F(1,22)=13.86$, $p<.01]$. Left and right composites of highly familiar faces were rated more similar to each other (mean $=7.0$ ) than were left and right composites of less familiar faces (mean $=6.6$ ). This small effect may reflect a greater ability to see that both composites look somewhat like the person for more familiar faces. There was no effect of expression $[F(1,22)<1]$ or order $[F(1,22)=1.22$, $p>.05]$. The only significant interaction was between order and expression (all other $F \mathrm{~s}<1)[F(1,22)=6.94$, $p<.05]$. For neutral faces, the order in which the neutral and smiling faces were seen did not affect the similarity ratings (mean $=6.75$, neutral then smiling faces; mean $=6.71$, smiling then neutral faces), whereas the smiling composites were rated more similar to each other when they were seen after the neutral faces (mean $=7.31$ ) than when they were seen first (mean $=6.40$ ).

A two-way analysis of variance with faces as the random factor, and expression type and familiarity as withinface factors, was carried out to determine whether the likeness results generalized across faces. ${ }^{12}$ The dependent variable was the proportion of subjects choosing the left composite as a better likeness for each face (see Table 4). The mean proportion of subjects choosing the left composite for each face was .60 , which was not significantly greater than $.50[F(1,19)=1.36, p>.05]$. There was no effect of expression type $[F(1,19)<1]$ or familiarity $[F(1,19)<1]$. Nor was there any interaction between expression and familiarity $[F(1,19)=3.39, p>.05]$. These results show that the bias in favor of left composites' looking more like the faces does not generalize across faces. It only occurs for a subset of the stimuli. In order to interpret this result, data were collected on which composite looked more like a real face (see control results below). Perhaps the left composites were chosen only if they looked more like a real face. The absence of a familiarity effect is consistent with this possibility, because it suggests that the judgments may have been based on some aspect of the faces that was independent of any correspondence with their memory representations.

Control results. Undergraduates who were unfamiliar with the faces were asked to decide which composite of each pair looked more like a real face (their data are sum- 
Table 4

Mean Proportion of Subjects Choosing the Left Composite as a Better Likeness for Each Face (Experiment 2)

\begin{tabular}{lllcc}
\hline & \multicolumn{4}{c}{ Familiarity } \\
\cline { 2 - 3 } Expression & Low (Rated 2 or 3) & & High (Rated 4 or 5) \\
\cline { 2 - 3 } \cline { 5 - 6 } Nean & SE & & Mean & SE \\
\hline Seutral & .63 & .06 & .56 & .05 \\
Smiling & .60 & .06 & .63 & .04 \\
\hline
\end{tabular}

marized in Table 3). The mean proportion of left composites chosen was .39, which was significantly less than $.50[F(1,22)=17.32, p<.001]$. The right composites looked more like real faces than did the left composites. ${ }^{13}$ Therefore, the bias in favor of left composites did not result from subjects' simply choosing the more realistic composite (but see below). There was no effect of order $[F(1,22)<1]$ or expression $[F(1,22)<1]$, and no interaction between the two $[F(1,22)=1.09, p>.05]$.

In order to determine how the realism of the composites influenced the likeness judgments, if at all, performance on these judgments (by subjects familiar with the faces) was reexamined as a function of which composite looked more like a real face (according to subjects who were unfamiliar with the faces). There was a significant bias to choose the left composite as more like the person (mean $=.73$ ) when that composite looked more like a real face $[t(23)=7.77, p<.001]$. However, when the right composite looked more like a real face, the proportion of left composites chosen (mean $=.54$ ) was not significantly greater than $.50[t(23)=1.20, p>.05]$. These results suggest that the left composites looked more like the person, but that this bias was competing with a bias to choose the composite that looked more like a real face. When the left composite satisfied both criteria (i.e., looking like the person and looking realistic) there was a large bias to choose it. However, when the composites each satisfied a different criterion, no clear bias emerged. It should be noted, however, that subjects were not simply choosing the more facelike composite, because this would have resulted in a bias in favor of the right composite when it looked more like the face (which did not occur).

\section{Emotion Judgments}

A three-way analysis of variance was carried out on the mean proportion of left composites chosen as expressing more emotion (see Table 5), with the same factors as for the likeness judgments analysis. There was a significant main effect of expression $[F(1,22)=12.00$, $p<.01]$. The proportion of left composites judged as expressing more emotion was higher for the smiling (mean $=.56$ ) than for the neutral faces (mean $=.46$ ), but neither proportion differed significantly from .50 $[F(1,22)=1.89, p>.05$ (neutral); $F(1,22)=2.82$, $p>.05$ (smiling)]. Nor was the overall proportion of left composites chosen $($ mean $=.51)$ significantly different from .50 $[F(1,22)<1]$. However, if one considers the proportion of left composites chosen for the neutral faces as a baseline of asymmetry of expressiveness, then the results indicate that smiles (relative to neutral expressions) were perceived to be more intensely expressed on the right hemiface. This is the opposite result to that normally found with unfamiliar faces. There were no significant effects of order $[F(1,22)<1]$ or familiarity $[F(1,22)<1]$. The only significant interaction was between familiarity and order $[F(1,22)=6.15, p<.05]$. When neutral faces were judged first, left composites were chosen more often for high- (mean $=.56$ ) than for low-familiarity faces (mean $=.44$ ). This was reversed when smiling faces were judged first (mean $=.47$, high-familiarity faces; mean $=.55$, low-familiarity faces). The mean ratings of expression for the composites chosen as showing more emotion were $3.8(\mathrm{SD}=.5)$ for the neutral composites, and $6.0(\mathrm{SD}=.4)$ for the smiling composites $(1=\mathrm{sad}, 7=$ happy).

An analysis of variance was carried out with faces as the random factor and the proportion of subjects choosing the left composite for each face as the dependent variable. Familiarity, expression type, and order were withinface factors. As in the analysis with subjects as a random factor, left composites were chosen more often for smiling (mean $=.52$ ) than for neutral faces (mean $=.47$ ). However, the effect of expression was not significant $[F(1,19)<1]$. Nor was the overall proportion of left composites chosen (mean $=.50$ ) significantly different from $.50[F(1,19)<1]$. There were no significant effects of familiarity [mean $=.48$, low-familiarity faces; mean $=.51$, high-familiarity faces; $F(1,19)<1]$ or order [mean $=.51$, neutral then smiling faces; mean $=$ .48 , smiling then neutral faces; $F(1,19)<1$ ]. There were no significant interactions. Thus the interaction between familiarity and order, found with subjects as a random factor, does not generalize across faces. Nor does the main effect of expression type generalize across faces, although the means are in the expected order (the large error variance prevented the difference from even approaching significance). These results suggest that faces may vary considerably in the degree to which the two sides express emotion, a result that has been reported by other researchers (Heller \& Levy, 1981). The reason for this variation is not known, and does not appear to be due to the handedness of the posers (Campbell, 1978; Heller \& Levy, 1981). One possible source of variation in the present results is that both spontaneous and posed smiles, which have different neural control mechanisms (Rinn, 1984), may have been included in the stimuli. Some

Table 5

Mean Proportion of Left Composites Chosen as Expressing More Emotion (Experiment 2)

\begin{tabular}{|c|c|c|c|c|c|c|}
\hline \multirow[b]{3}{*}{ Expression } & \multicolumn{6}{|c|}{ Familiarity } \\
\hline & \multicolumn{2}{|c|}{ Unfamiliar } & \multicolumn{2}{|c|}{ Low (Rated 2 or 3 ) } & \multicolumn{2}{|c|}{ High (Rated 4 or 5 ) } \\
\hline & Mean & SE & Mean & $\overline{\mathrm{SE}}$ & Mean & SE \\
\hline Neutral & .46 & .02 & .43 & .04 & .48 & .03 \\
\hline Smiling & .45 & .02 & .56 & .04 & .55 & .03 \\
\hline
\end{tabular}

Note-Data for the unfamiliar faces are from different subjects than data for the low- and high-familiarity faces. 
authors have claimed that asymmetries in expressiveness only occur for posed smiles (Ekman, Hager, \& Friesen, 1981), whereas others have reported left facedness for spontaneous smiles (Borod, Koff, \& White, 1983, for males only; Heller \& Levy, 1981; Moscovitch \& Olds, 1982). None of these studies, however, has assessed the effect of familiarity of the faces on judged asymmetries in expressiveness.

Control results. In order to determine whether the unexpected bias to judge left composites (right hemifaces) as more expressive (at least for some faces) was due to the familiarity of the faces rather than to some idiosyncracy of the stimuli used, data were collected from control subjects who were unfamiliar with the faces (see Table 5). The overall mean proportion of left composites chosen as more expressive was .45 , which was significantly less than $.50[F(1,22)=3.82, p<.05$, onetailed], indicating a bias in favor of left hemifaces (right composites), as is normally found for unfamiliar faces. Thus, it appears that the bias in favor of left composites only occurs if the faces are familiar. However, if the bias for unfamiliar faces (mean $=.45$ ) was assessed relative to the baseline established by neutral faces (mean $=.46$ ), then there was no asymmetry $[F(1,22)<1]$. For the control subjects, there was no effect of order $[F(1,22)=1.37$, $p>.05$; mean $=.47$, neutral then smiling faces; mean $=.43$, smiling then neutral faces], or expression $[F(1,22)<1$; mean $=.46$, neutral; mean $=.45$, smiling]. Nor was there a significant interaction between order and expression $[F(1,22)<1]$.

These results suggest that familiarity with a face may affect the perception of which hemiface is more expressive. When the faces were unfamiliar, there was no difference in which hemiface appeared more expressive. However, when the (same) faces were familiar, there was a bias in favor of the left composites (right hemifaces). These results were calculated relative to a baseline asymmetry established by the neutral faces. If, however, the asymmetry was calculated by comparison with a .50 baseline, then there was an asymmetry in favor of right composites (left hemifaces) when the faces were unfamiliar (as is normally found), but no asymmetry when they were familiar. The variation in results as a function of the baseline asymmetry used, together with variability across faces, makes it difficult to draw firm conclusions about asymmetries in the expression of emotion. However, the results do suggest quite clearly that familiarity with a face affects the perception of asymmetries in emotional expression.

The finding that right hemifaces (left composites) of familiar faces appear more expressive may be due to the perceptual bias in favor of the half-face on the observer's left. The right hemiface has always appeared on the left of a familiar face, and people may have learned to interpret the expression on that hemiface better than that on the left hemiface. This seems particularly likely considering that the right hemiface would have fallen mostly in the left visual field and been projected directly to the right hemisphere, which is better at perceiving emotional expressions (Bryden \& Ley, 1983). Thus, the right hemiface of a familiar face could be perceived as showing more emotion, even though the left hemiface might in fact be exhibiting a greater degree of movement relative to some neutral baseline expression (Borod et al., 1983). The use of direct measurement to assess asymmetries in the expressiveness of the two sides of the face assumes that there is a causal connection between amount of muscle movement and degree of expressiveness (Borod et al., 1983; Ekman et al., 1981; Lynn \& Lynn, 1938, 1943). However, the type of movement may be as important as the amount, and the effect of both may be modified by other factors such as how familiar the face is (as suggested by the present results), how genuine the expression looks, or even how asymmetric the face is. The effect of other factors, such as attitudes toward the person depicted and the subject's own mood (see Bower \& Cohen, 1982), on the perception of facial expressions and asymmetries in expressiveness may also be important future research topics.

\section{GENERAL DISCUSSION}

The results of Experiments 1 and 2 provide converging evidence that subtle configural information about the left-right organization of a face is encoded in naturally acquired memory representations of faces. In Experiment 1 , subjects were able to discriminate between normal and mirror-reversed photographs of familiar faces. They regarded the normal photographs as better likenesses, and this did not depend on the presence of single, asymmetrically located features such as hair partings, moles, or warts. In Experiment 2, left composites were regarded as better likenesses than right composites, despite the fact that this bias appeared to compete with one in favor of the more realistic of the two composites. The left composite is normally regarded as a better likeness than the right composite when the two are compared with a photograph of the real face. The results of Experiment 2 indicate that this asymmetry in the perceptual representation is retained in memory and, therefore, provides further evidence that left-right asymmetries are encoded in memory representations of faces. The fact that such configural information is encoded suggests that models of face representations as sets of individual features (eyes, nose, mouth, hair, moles, etc.) are inadequate. Rather, configural information about the spatial relations between these features also appears to be represented, and may be an important determinant of a face's appearance.

There is a theoretical distinction between the kind of information that is represented (content) and how information is represented (structure). The experiments reported here were designed to determine whether a particular kind of configural information is represented in memory. Although the content and structure issues are logically distinct, the kind of structure that is most effective for a given representation depends on the informa- 
tion to be represented and the functions to be fulfilled by the representation (Marr, 1982). In the case of faces, if the only information encoded concerned individual features, a feature list might be considered an adequate representational structure. However, this conclusion is challenged by several findings. Davies and Christie (1982) have shown that subjects find it difficult to match stored impressions of faces to isolated features. Homa, Haver, and Schwartz (1976) have also shown that recognition of individual features is better for normal than for scrambled faces, and Davies, Ellis, and Shepherd (1977) found that subjects had difficulty discriminating between features (e.g., two different noses) when they were embedded in the same face. These results suggest that even information about individual features may not be stored independently of other information about the face. This should not be surprising when one considers that the function of such a representation is to subserve recognition of the face as an integrated unit. Analysis of the face into independent component features seems unsuited to such a task. Configural information, which the present results demonstrate is also represented, seems especially unsuited to representation in an analytic structure, such as a feature list, although configural information can, in principle, be represented analytically (Garner, 1981). A more natural and heuristically useful alternative would be that faces are represented in a holistic or analogical representation, whose structure directly mirrors the spatial properties of the face.

The issue of how increasing familiarity changes one's mental representation of a face and one's perception of that face has received relatively little attention. Yet introspection suggests that with increasing familiarity, a face may come to look quite different from one's initial impression of it, especially if the change in familiarity is accompanied by a change in emotional response to the person. A second aim of the present experiments was to investigate perceived asymmetries in the emotional expressiveness of the two sides of the face in familiar faces, and to determine whether these were the same as normally found for unfamiliar faces. Perceived asymmetries in the amount of emotion displayed in the two sides of a face were found to depend on both the familiarity of the face and the baseline against which the asymmetry was measured. The right hemiface was judged more expressive for smiling familiar faces, relative to a neutral baseline expression, but not to a .50 baseline. Subjects who were unfamiliar with the faces judged the left hemiface to be more expressive relative to a .50 baseline (the usual result), but there was no asymmetry relative to the neutral expression asymmetry. This variability makes it difficult to draw conclusions about asymmetries in the expressiveness of faces. However, the results do indicate that perceived asymmetries in the expressiveness of the two hemifaces depend on the familiarity of the faces. This suggests that mental representations of faces are dynamic structures that change with increasing familiarity of a face. Therefore, the results of experiments based on minimal exposure to faces (the typical paradigm) may have serious limitations for understanding how faces are normally represented in memory.

Most research on face perception and recognition has focused on the contribution of individual features to recognition (see Shepherd et al., 1981, for an excellent review of these studies). Less attention has been given to the role of configural information, which is harder to manipulate experimentally. It is hoped that the present experiments will help to counteract this imbalance and lead to more realistic models of face representations.

\section{REFERENCES}

BADDELEY, A. D. (1979). Applied cognitive and cognitive applied psychology: The case of face recognition. In L. G. Nilsson (Ed.), Perspectives on memory research. Hillsdale, NJ: Erlbaum.

Bahrick, H. P., Bahrick, P. O., \& Wittlinger, R. P. (1975). Fifty years of memory for names and faces: A cross-sectional approach. Journal of Experimental Psychology: General, 104, 54-75.

BoroD, J. C., KoFF, E., \& WHITE, B. (1983). Facial asymmetry in posed and spontaneous expressions of emotion. Brain \& Cognition, 2 , $165-175$

BOWER, G. H., \& COHEN, P. R. (1982). Emotional influences in memory and thinking: Data and theory. In M. S. Clark \& S. T. Fiske (Eds.), Affect and cognition. Hillsdale, NJ: Erlbaum.

BOWER, G. H., \& KARLIN, M. B. (1974). Depth of processing pictures of faces and recognition memory. Joumal of Experimental Psychology, 103, 751-757.

Bradshaw, J. L., \& Sherlock, D. (1982). Bugs and faces in the two visual fields: The analytic/holistic processing dichotomy and task sequencing. Cortex, 18, 211-226.

Brodal, A. (1965). The cranial nerves. Oxford: Blackwell.

BRydeN, M. P., \& LEY, R. G. (1983). Right-hemispheric involvement in the perception and expression of emotion in normal humans. In K. M. Heilman \& P. Satz (Eds.), Neuropsychology of human emotion. New York: The Guilford Press.

CAMpBell, R. (1978). Asymmetries in interpreting and expressing a posed facial expression. Cortex, 14, 327-342.

Courtors, M. R., \& Mueller, J. H. (1979). Processing multiple physical features in facial recognition. Bulletin of the Psychonomic Society, 14, 74-76.

Craik, F, I. M., \& LockharT, R. S. (1972). Levels of processing: A framework for memory research. Journal of Verbal Learning \& Verbal Behavior, 11, 671-684.

Davies, G. M., \& ChRISTIE, D. (1982). Face recall: An examination of some factors limiting composite production accuracy. Journal of Applied Psychology, 67, 103-109.

Davies, G. M., Ellus, H. D. , \& Shepherd, J. W. (1977). Cue saliency in faces as assessed by the Photofit technique. Perception, 6, 263-269.

Ekman, P., Hager, J. C., \& Friesen, W. V. (1981). The symmetry of emotional and deliberate facial actions. Psychophysiology, 18, 101-106.

ELLIS, H. D. (1983). The role of the right hemisphere in face perception. In A. W. Young (Ed.), Functions of the right cerebral hemisphere. London: Academic Press.

GARNER, W. R. (1981). The analysis of unanalyzed perceptions. In M. Kubovy \& J. R. Pomerantz (Eds.), Perceptual organization. Hillsdale, NJ: Erlbaum.

GILBERT, C., \& BAKAN, P. (1973). Visual asymmetry in perception of faces. Neuropsychologia, 11, 355-362.

Harmon, L. D. (1973). The recognition of faces. Scientific American, 229, 70-82.

Heller, W., \& Levy, J. (1981). Perception and expression of emotion in right-handers and left-handers. Neuropsychologia, 19, 263-272.

Homa, D., Haver, B., \& Schwartz, T. (1976). Perceptibility of schematic face stimuli: Evidence for a perceptual Gestalt. Memory \& Cognition, 4, 176-185. 
Kolb, B., Milner, B., \& Taylor, L. (1983). Perception of faces by patients with localized cortical excisions. Canadian Journal of Psychology, 37, 8-18.

Levy, J., Trevarthen, C., \& Sperry, R. W. (1972). Perception of bilateral chimaeric figures following hemispheric deconnexion. Brain, 95, 61-78.

Lindzey, G., Prince, B., \& Wright, W. K. (1952). A study of facial asymmetry. Journal of Personality, 21, 68-84.

LYNN, J. G., \& LYNN, D. R. (1938). Face-hand laterality in relation to personality. Journal of Abnormal Psychology, 33, 291-332.

LYNN, J. G., \& LYNN, D. R. (1943). Smile and hand dominance in relation to basic modes of adaptation. Journal of Abnormal \& Social Psychology, 38, 250-276.

MarR, D. (1982). Vision. San Francisco: Freeman.

MCCURDY, H. G. (1949). Experimental notes on the asymmetry of the human face. Journal of Abnormal \& Social Psychology, 44, 553-555.

McKelvie, S. J. (1983). Effects of lateral reversal on recognition memory for photographs of faces. British Journal of Psychology, 74, 391-407.

Mita, T. H., Dermer, M., \& Knight, J. (1977). Reversed facial images and the mere-exposure hypothesis. Joumal of Personality \& Social Psychology, 35, 597-601.

Moscovitch, M., \& OLDs, J. (1982). Asymmetries in emotional facial expressions and their possible relation to hemispheric specialization. Neuropsychologia, 20, 71-81.

RHODEs, G. (1985a). Lateralized processes in face recognition. British Journal of Psychology, 76, 249-271.

RHodes, G. (1985b). Perceptual asymmetries in face recognition. Brain \& Cognition, 4, 197-218.

RINN, W. E. (1984). The neuropsychology of facial expressions: A review of the neurological and psychological mechanisms for producing facial expressions. Psychological Bulletin, 95, 52-77.

SERGENT J. (1984a). Configural processing of faces in the left and right cerebral hemispheres. Journal of Experimental Psychology: Human Perception \& Performance, 10, 554-572.

SERGENT, J. (1984b). An investigation into component and configural processes underlying face perception. British Journal of Psychology, $75,221-242$.

ShePHERD, J., DAvies, G. , \& Ellis, H. (1981). Studies of cue saliency. In G. Davies, H. Ellis, \& J. Shepherd (Eds.), Perceiving and remembering faces. New York: Academic Press.

WARrington, E. K., \& ACKRoYD, C. (1975). The effect of orienting tasks on recognition memory. Memory \& Cognition, 3, 140-142.

WINOGRAD, E. (1978). Encoding operations which facilitate memory for faces across the life span. In M. M. Gruneberg, P. E. Morris, \& R. N. Sykes (Eds.), Practical aspects of memory. New York: Academic Press.

WiNOGRAD, E. (1981). Elaboration and distinctiveness in memory for faces. Journal of Experimental Psychology: Human Learning \& Memory, 7, 181-190.

WolfF, W. (1933). The experimental study of forms of expression. Character \& Personality, 2, 168-176.

\section{NOTES}

1. Ellis (1983) has pointed out, however, that Winograd (1981) does not have the control group (no special encoding instructions) necessary to determine whether elaboration improves performance or whether scan- ning for a single (nondistinctive) feature impairs performance, relative to some baseline level.

2. The left composite is made up of the left half of the face (from the viewer's perspective) reflected about the midline, and the right composite is made up of the right half reflected about the midline. If the two sides of a face are denoted $L$ and $R$, and the mirror images of these are denoted $L^{\prime}$ and $R^{\prime}$, respectively, then the left composite is $L L^{\prime}$ and the right composite is $R^{\prime} R$.

3. I will use the term half-face to refer to the side of the face from an observer's point of view, and the term hemiface to refer to the side of the face from its owner's point of view.

4. It should be noted that the phenomenon of facedness appears to be independent of this bias in favor of the left half-face.

5. Selection was based on quality of the photographs. Two of the men and 5 of the women were photographed from a distance that gave a head-and-shoulders picture, rather than a head-only picture. Removing these from the set left 9 male and 11 female head-only picture pairs. One of the head-and-shoulders male pairs was used in order to get 10 male picture pairs. None of the head-and-shoulders female pairs was used. One of the women in the set of 11 usable picture pairs was a visitor to the department and was not known by many students, so her pictures were not used.

6. Collapsing the familiarity categories in this way reduced the average number of missing data per cell from 3.5 to 2.0 out of 20 . Data were missing if a subject did not assign any of the faces to a particular familiarity level. In this and all subsequent analyses of variance reported, missing data were replaced by the mean score for that cell.

7. All faces were classified as low familiarity by some subjects and as high familiarity by others.

8. Only 4 of the 7 posers in the self-judgments part had asymmetric hairstyles.

9. Pilot testing indicated that subjects who were familiar with the faces found it difficult to make these judgments without being biased to choose the composite that looked more like the actual person.

10 . This assumes that the asymmetry in favor of the side of the face appearing on the viewer's left looking more like the face is retained in memory. This assumption is confirmed by the results of the likeness judgments in Experiment 2

11. As in Experiment 1, the completely unfamiliar faces, rated 1 on the familiarity scale, were not included in the scores, and the four remaining familiarity levels were collapsed into two levels-low and high. There were no significant differences between scores in the collapsed categories $[F(1,66)<1$, familiarity levels 2 (mean $=.70$ ) and 3 (mean $=.63$ ) $F(1,66)<1$, familiarity levels 4 (mean $=.61$ ) and 5 (mean $=.63)]$. Order was not included as a factor in the table because it did not yield any significant effects.

12. Order was not included as a factor because it was not a theoretically important variable, and it did not have a significant effect or any significant interactions in the analysis with subjects as the random factor.

13. Casual inspection of the composites revealed that for 7 of the 10 pairs that showed clear differences in face width, the right composite face was wider than the left composite face. This was true for both the neutral and smiling picture pairs. It is possible that the wider faces looked more natural than the narrower faces.

(Manuscript received May 16, 1985; revision accepted for publication October 21,1985 .) 